\title{
Enhancing the yield of high-order harmonics with an array of gas jets
}

\author{
Angela Pirri ${ }^{1}$, Chiara Corsi $^{1}$ and Marco Bellini ${ }^{1,2}$ \\ ${ }^{1}$ LENS, Via Nello Carrara 1, 50019 Sesto Fiorentino, Florence, \\ Italy; ${ }^{2}$ Istituto Nazionale di Ottica Applicata, Largo E. Fermi 6, I-50125 Firenze, Italy
}

(Dated: October 24, 2018)

\begin{abstract}
We report the experimental observation of an enhancement in the yield of high-order harmonics using an array of gas jets as the source medium. By comparing the experimental outcome for jet arrays of different spacings with the predicted harmonic intensity in the case of slit sources of equivalent lengths, we clearly show how the periodic modulation of the gas density can improve the harmonic yield. This behavior may attributed to a quasi-phase-matching effect which increases the length of coherent harmonic build-up during propagation by partially counteracting the dephasing induced by free electrons.
\end{abstract}

PACS numbers: $32.80 . \mathrm{Rm}, 42.65 . \mathrm{Ky}, 32.80 . \mathrm{Fb}$

Keywords:

Since its discovery in 1987 [1], the phenomenon of highorder harmonic generation (HHG) has become one of the most interesting topics in the field of highly nonlinear processes. Apart from its fundamental physics interest, HHG is now one of the most promising ways to obtain tunable, short-pulse, narrow-band radiation in the vacuum and extreme ultraviolet (VUV and XUV), and in the soft X-ray regions, where other coherent sources are scarcely available. However, the possibility of using high-order harmonics as a table-top VUV-XUV coherent source for applications is strongly connected to the optimization of the brightness of the source over the broadest spectral range.

As in many other nonlinear processes, conversion efficiency in HHG depends on the interplay between the single-atom response [2] and the macroscopic response during propagation in the medium [3]. In particular, a constructive interference of the harmonic field emitted from different locations of the source length can only be obtained over the so-called coherence length $L_{c}$. For media longer than $L_{c}$, destructive interference soon depletes the generated field and dramatically limits the conversion efficiency. By an appropriate choice of the interaction parameters it is often possible to make the coherence length longer than the medium, thus reaching the so-called phase-matching conditions. However, when phase-matching is not achievable, one can still artificially beat the coherence length limits by properly modulating the interaction parameters along the field propagation direction. Examples of such quasi-phase-matching (QPM) techniques now abound for low-order nonlinear phenomena in structured crystals, and a few examples have been recently demonstrated in HHG. Many different phenomena contribute to limit the coherence length in HHG. Here, the atomic dispersion and the geometric Guoy phase [4, 5], are always accompanied by the dispersion connected to free electrons in the partially ionized medium [6, 7], and by the characteristic atomic dipole phase [8, 9, 10, 11, 12]. Depending on the gas type and density, and on the level of ionization, the coherence length in HHG is usually so short as to pose a serious limit to significant conversion efficiencies. Differently from QPM in periodically-poled nonlinear crystals 13], where the sign of the nonlinear coefficient is periodically flipped so as to always add constructively interfering contributes to the generated field, QPM in HHG has so far been demonstrated by periodically switchingoff harmonic generation during the out-of-phase intervals. Either a modulation of the pump laser intensity in a modulated-diameter, hollow-core, gas-filled waveguide 14, 15, 16], or the use of a counter-propagating train of pulses [17, 18] were used at this purpose. Recently, theoretical studies have predicted the possibility of achieving QPM by periodically modulating the density of the gas medium [19, 20], and a proof-of-concept work has shown the coherent buildup of the harmonic field in two separated gas sources [21].

Here we introduce a simple scheme for the generation of high-order harmonics from a whole array of arbitrarilyspaced gas sources. We compare the experimental results to those one may expect from a single-slit source of equivalent length and show that, for particular spacings and harmonic orders, a clear enhancement of the yield is observed that may be attributed to at least a partial QPM effect. Figure 1 shows a schematic of the experimental

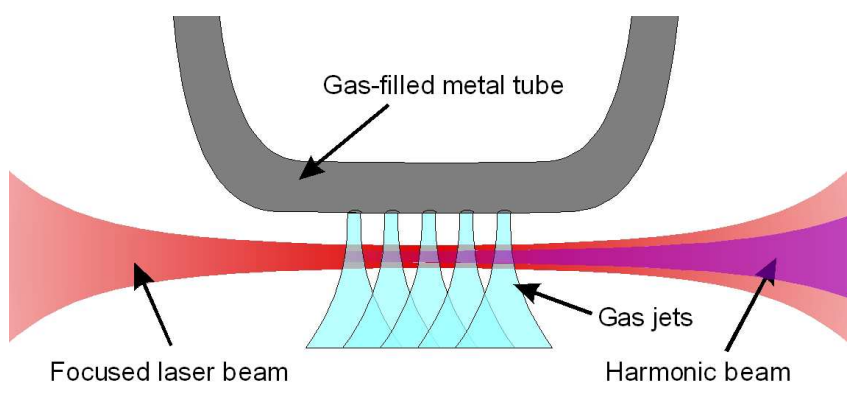

FIG. 1: Scheme of the interaction region for the generation of high-order harmonics. An array of laser-drilled holes in a gas-filled metal tube produces a periodic modulation of the medium density encountered by the focused pump laser pulses. 
setup. The gas jets were obtained by laser drilling a linear array of equally-spaced holes, each with a diameter of about $30 \mu \mathrm{m}$, over a total distance of about $2 \mathrm{~mm}$ in a metal tube. The distance between the holes depends on their number: we used tubes with three, five, ten and twenty holes, corresponding to separations of about $600,400,200$ and $100 \mu \mathrm{m}$, respectively. The $30-\mathrm{fs}$ pulses from an amplified Ti:Sapphire laser were focused by a 50-cm focal-length lens directly below the hole array while the tube was filled with xenon at low pressure. The peak intensity in the focal spot, with a beam waist of $30 \mu \mathrm{m}$ and a corresponding confocal parameter of $5 \mathrm{~mm}$ (chosen to be longer than the global source length), was about $5.0 \times 10^{14} \mathrm{~W} / \mathrm{cm}^{2}$, well above the saturation intensity of xenon. Intense plasma light emission was clearly observed from the interaction zones under the tube holes (see Fig.2). The gas backing pressure in the tube was regulated in order to maintain a constant density (estimated to be about $6.6 \times 10^{17}$ atoms $/ \mathrm{cm}^{3}$ and chosen according to the maximum pressure allowed by the pumping system under continuous flow conditions) in the interaction region of each gas jet for the different arrays. A single hole configuration operated at the same gas density was used as a reference. The hole array was aligned to the laser propagation direction and the tube was mounted on a translation stage allowing for precise positioning with respect to the laser focus. The spectrum of the emitted harmonics was dispersed by a Pt-Ir normal incidence spherical grating (600 lines/mm) and detected by a phosphor screen (converting the XUV-photons into visible light) and a photomultiplier.

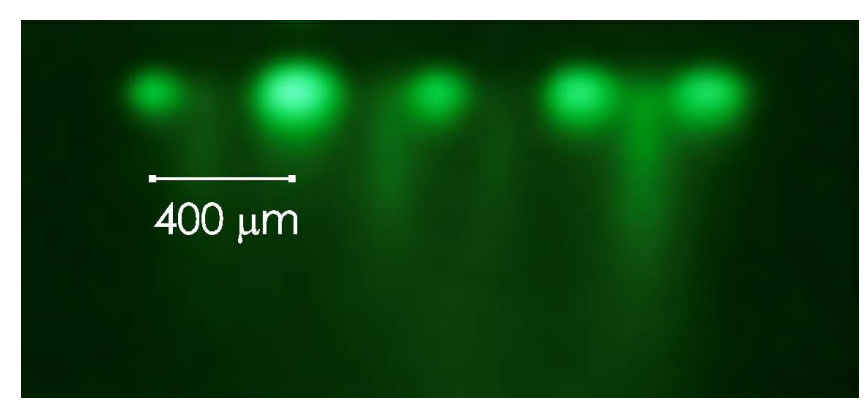

FIG. 2: Picture of the harmonic generation region in the 5hole gas jet configuration; the plasma light generated by intense laser ionization is evident in correspondence with the gas jets, separated by about $400 \mu \mathrm{m}$.

In order to clearly bring to evidence the effects of the gas jet array and to eliminate all systematic contributions to the detected number of XUV photons (like the different reflectivity of the grating or efficiency of the phosphor screen at different wavelengths), all the harmonic yields obtained in the multiple-source configurations were normalized to the respective single-source ones. What we obtain for each harmonic order is thus the effective enhancement factor due to the presence of the source array. This enhancement factor can be simply compared to the theoretical expectation for a single slit source emitting gas at the same density and with a length equivalent to the sum of the individual sources in the array. Two limit situations are considered in this case: in the first one, the dispersion effects due to the presence of free electrons are properly accounted for, limiting the coherence length of the nonlinear interaction; in the second one, an infinite coherence length, corresponding to an unrealistic perfect phase-matching, is assumed. The expected dependence of the harmonic yield as a function of the interaction length $L$, the absorption length $L_{a}$, and coherence length $L_{c}$ is estimated according to [22]:

$n_{p}\left(L, L_{a}, L_{c}\right) \propto \frac{L_{a}^{2}}{1+4 \pi^{2}\left(\frac{L_{a}}{L_{c}}\right)^{2}}\left[1+e^{-\frac{L}{L_{a}}}-2 \cos \left(\pi \frac{L}{L_{c}}\right) e^{-\left(\frac{L}{2 L_{a}}\right)}\right]$

and the corresponding enhancement factor for each harmonic order $q$ is simply given by:

$$
E_{q}\left(n, L_{a_{q}}, L_{c_{q}}\right)=\frac{n_{p}\left(n \cdot L, L_{a_{q}}, L_{c_{q}}\right)}{n_{p}\left(L, L_{a_{q}}, L_{c_{q}}\right)}
$$

where $n$ is the number of elementary sources, whereas $L_{a_{q}}$ and $L_{c_{q}}$ are the absorption and coherence lengths calculated for each harmonic order by taking into account the source gas density and always considering a full ionization of the medium [23]. Since we are working in a relatively loose focus configuration, ionization-related dispersion is the only factor taken into account for the estimation of the coherence length. The medium length $L$ for a single gas jet source has been fixed to $45 \mu \mathrm{m}$. Note that the single-source length $L$ and the gas density are not independent and only their product matters in the above estimations: increasing the source length has the same effect as increasing the gas pressure by the same factor.

Simple considerations can be made before looking at the experimental data. First of all, if both absorption and phase mismatch were absent, then the enhancement would be independent of the harmonic order and proportional to the square of the medium length thanks to the constructive interference of all emitters. In other words, one should expect an enhancement factor just equal to the square of the number $n$ of sources in the array. Rather interestingly, such behavior is almost respected for our lowest-order harmonics. In the case of the fifth and seventh harmonics, which are just below the single-photon ionization threshold of xenon, a low absorption and a relatively long coherence length contribute to reach high experimental enhancement factors, up to about 18, or about $70 \%$ of the ideal case for the fifth harmonic in the 5-hole array (see Fig.3b). Enhancement factors up to 35 were observed for the fifth harmonic also in the 10 and 20-hole configurations (Figs.3c and 3d), but in these cases the ideal absorption- and mismatch-free enhancements would have been much higher.

When absorption processes are turned on, the maximum enhancement factor decreases from the ideal value of $n^{2}$, and starts to strongly depend on the harmonic order. The dotted curves in Fig.3 show this expected behavior, with the pronounced dip due to absorption for 

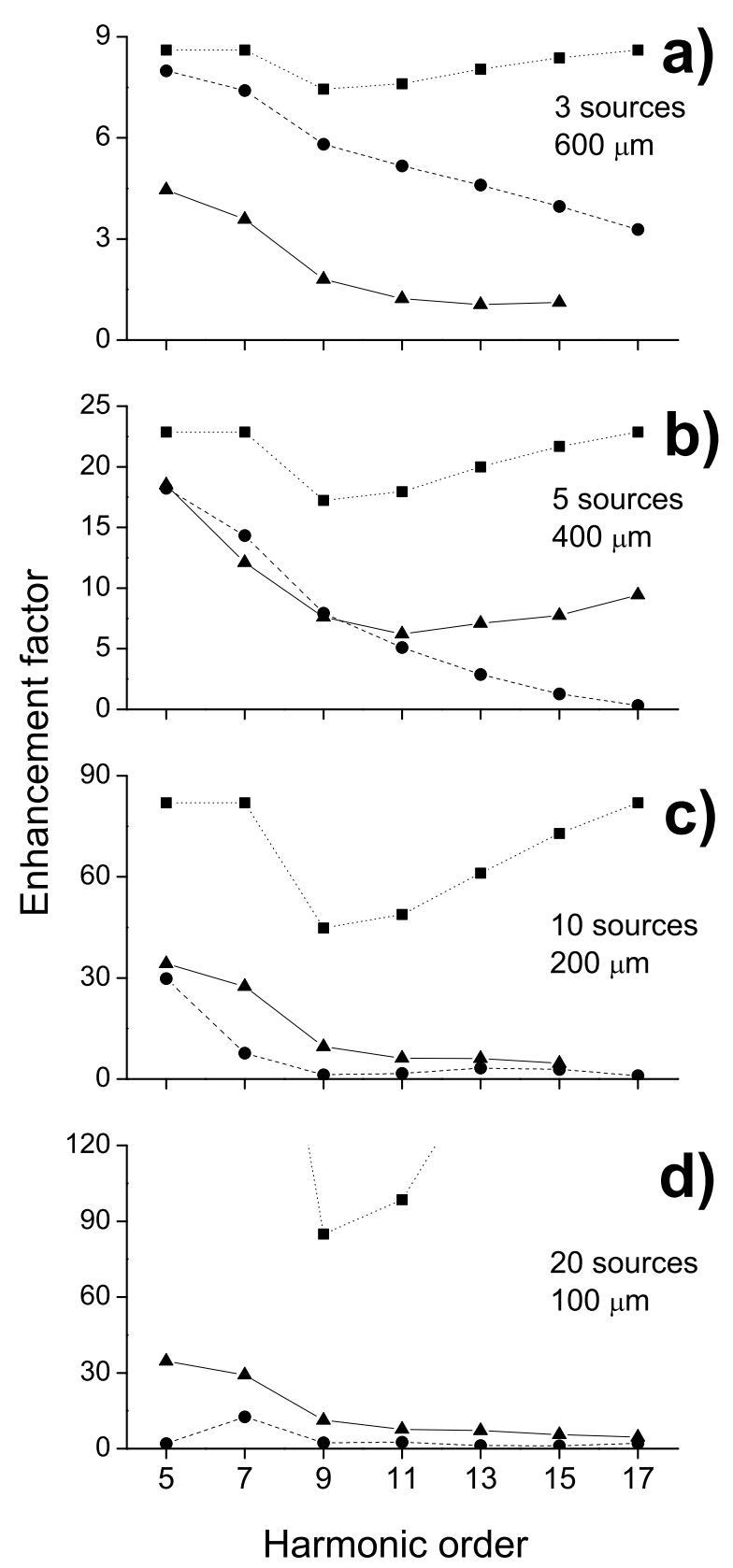

FIG. 3: Harmonic yield enhancement factors for different gasjet source arrays normalized to the single source configuration. Solid curves: experimental data; dotted curves: calculated data for a single slit source of equivalent length in the case of perfect phase-matching; dashed curves: calculated data for a single slit source including phase mismatch effects.

harmonics just above the ionization threshold. Finally, when phase mismatch is considered, a strong suppression of higher-order harmonics and wild oscillations for lower orders are expected (dashed curves in Fig.3).

Experimental results are presented in Fig.3 (solid curves) for the four array configurations together with the calculated curves for the long slit case, with and without dispersion effects. In most of the cases for the 10 and 20-hole arrays (corresponding to source spacings of 200 and $100 \mu m$, respectively), the experimental points stay well above the curves corresponding to the non-phasematched situation. For the 5-hole array $(400 \mu \mathrm{m}$ spacing), the expected mismatched behavior is exactly followed by the lower orders, whereas a clear departure from this trend is obtained above the $11^{t h}$ harmonic. In particular, a 30-fold increase of the enhancement factor is obtained for the $17^{\text {th }}$ harmonic, which should have been severely suppressed by a limited coherence length in the mismatched situation. Although the enhancement levels expected for a completely phase-matched situation are never reached, the observed behaviors suggest that at least a partial quasi-phase-matching effect is present.

The experimental points for the 3-hole array have a similar trend as the predicted mismatched situation, but are always lower by a factor of about 2 . Besides indicating that no QPM effect is probably present in this case, the low enhancement factor may indicate some imperfect alignment of the rather distant (600 $\mu \mathrm{m}$ spacing) holes along the laser direction, or some strong effect of beam defocusing in the heavily ionized medium [7]. Although a similar phenomenon should be present also in the other array configurations, the closer spacing of the sources and a partial QPM might be able to counteract it.

As a final check, we also repeated the above measurements while varying the relative position between the gas source arrays and the laser focus in a $5 \mathrm{~mm}$ range around the optimum. No significant deviations from the above results were observed in such conditions, showing that the (position-insensitive) free-electron contribution to dispersion is the most important in these cases and can be effectively counteracted independently of the position.

In conclusion, the use of a periodically-modulated gas source has produced a clear enhancement in the yield of high-order harmonics. Such an enhancement, dependent on the harmonic order and on the period of the gas-density modulation, exceeds that predicted for a single slit source of equivalent length where phase-matching effects, mostly due to the presence of free electrons, are present. This strongly suggests a quasi-phase-matching effect connected to the periodicity along the propagation axis. The experimental scheme, using an array of gas jets operating in a continuous flow, is extremely simple and can be easily extended to longer interaction lengths or closer source spacings in order to further increase the harmonic yield or enhance shorter wavelengths. Additional analysis is in progress, but these results already show that a selective enhancement of the source brightness can be achieved in order to optimize specific applications. 
[1] A. McPherson, G. Gibson, H. Jara, U. Johann, T. S. Luk, I. McIntyre, K. Boyer, and C. K. Rhodes, J. opt. soc. Am. 4, 595 (1987); M. Ferray, A. L'Huillier, X. F. Li, L. A. Lompré, G. Mainfray, and C. Manus, J. Phys. $B$ 21, L31 (1988).

[2] P. B. Corkum, Phys. Rev. Lett. A 71, 1994 (1993).

[3] F. Reintjes, Non-Linear Optial Parametric Processes in Liquid and Gases, Academic Press, New York (1984).

[4] A. L'Huillier, X. Li, L. Lompre, J. Opt. Soc. Am. B 7, 527 (1990).

[5] A. L'Huillier, K. J. Schafer, and K. C. Kulander, Phys. Rev. Lett. 66, 2200 (1991).

[6] C. Altucci, T. Starczewski, E. Mevel, C.-G. Wahlström, B. Carré and A. LHuillier, J. Opt. Soc. Am. B 13, 148 (1996).

[7] M. Bellini, C. Corsi, and M. C. Gambino, Phys. Rev. A 64, 023411 (2001).

[8] M. Lewenstein, P. Salieres and A. L'Huillier, Phys. Rev. A 52, 4747 (1995).

[9] M. Bellini, C. Lyngå, A. Tozzi, M. B. Gaarde, T. W. Hänsch, A. L'Huillier and C.-G. Wahlström, Phys. Rev. Lett. 81, 297 (1998).

[10] C. Lyngå, M. B. Gaarde, C. Delfin, M. Bellini, T. W. Hänsch, A. L'Huillier and C.-G. Wahlström, Phys. Rev. A 60, 4823 (1999).

[11] C. Corsi, A. Pirri, E. Sali, A. Tortora and M. Bellini, Phys. Rev. Lett. 97, 023901 (2006).

[12] P. Salières, B. Carré, L. Le Déroff, F. Grasbon, G. G. Paulus, H. Walther, R. Kopold, W. Becker, D. B. Milosevic, A. Sanpera and M. Lewenstein, Science 292, 902 (2001).
[13] J. A. Armstrong, N. Bloembergen, J. Ducuing, and P. S. Pershan, Phys. Rev. A 127, 1918 (1962).

[14] I. P. Christov, H. C. Kapteyn and M. M. Murnane, Opt. Exp. 7, 362 (2000).

[15] E. A. Gibson, A. Paul, N. Wagner, R. Tobey, D. Gaudiosi, S. Backus, I. P. Christov, A. Aquila, E. M. Gullikson, D. T. Attwood, M. M. Murnane and H. C. Kapteyn Science 302, 95 (2003).

[16] A. Paul, R. A. Bartels, R. Tobey, H. Green, S. Weiman, I. P. Christov, M. M. Murnane, H. C. Kapteyn, S. Backus, Nature 421, 51 (2003).

[17] X. H. Zhang, A. L. Lytle, T. Popmintchev, X. B. Zhou, H. C. Kapteyn, M. M. Murnane, and O. Cohen, Nature Phys. 3, 270 (2007).

[18] S. L. Voronov, I. Kohl, J. B. Madsen, J. Simmons, N. Terry, J. Titensor, Q. Wang, and J. Peatross, Phys. Rev. Lett. 87, 133902 (2001).

[19] P. L. Shkolnikov, A. Lago and A.E. Kaplan, Phys. Rev. A 50, R4461 (1994).

[20] T. Auguste, B. Carré, and P. Salières, Phys. Rev. A 76, 011802(R) (2007).

[21] J. Seres, V. S. Yakovlev, E. Seres, CH. Streli, P. Wobrauschek, CH. Spielmann and F. Krausz, Nature Phys. 3, 878 (2007).

[22] E. Constant, D. Garzella, P. Breger, E. Mével, C. Dorrer, C. Le Blanc, F. Salin, and P. Agostini, Phys. Rev. Lett. 82, 1668 (1999).

[23] W. F. Chan, G. Cooper, X. Guo, G.R. Burton and C.E. Brion, Phys. Rev. A 46, 149 (1992). 